\title{
BMJ Open Randomised controlled trial of the impact of haemodiafiltration on uraemic neuropathy: FINESSE study protocol
}

\author{
Brendan Smyth, ${ }^{1}$ Arun V Krishnan, ${ }^{2}$ Martin Gallagher, ${ }^{1,3}$ Matthew Kiernan, ${ }^{4}$ \\ Paul Snelling, ${ }_{5}^{5}$ Carmel Hawley, ${ }^{6}$ Mangalee Fernando, ${ }^{9}$ Samantha Hand, ${ }^{3}$ \\ Kim Grimley, Jenny Burman, ${ }^{5}$ Anne Heath, ${ }^{7}$ Amy Kang, ${ }^{1}$ Vlado Perkovic, ${ }^{1}$ \\ Meg J Jardine ${ }^{1,3,7}$
}

To cite: Smyth B, Krishnan AV, Gallagher M, et al. Randomised controlled trial of the impact of haemodiafiltration on uraemic neuropathy: FINESSE study protocol. BMJ Open 2019;9:e023736. doi:10.1136/ bmjopen-2018-023736

- Prepublication history for this paper is available online. To view these files, please visit the journal online (http://dx.doi org/10.1136/bmjopen-2018023736).

Received 25 April 2018

Revised 4 September 2018 Accepted 30 November 2018

Check for updates

(c) Author(s) (or their employer(s)) 2019. Re-use permitted under CC BY-NC. No commercial re-use. See rights and permissions. Published by BMJ.

For numbered affiliations see end of article.

Correspondence to

Dr Brendan Smyth;

bsmyth@georgeinstitute.org.au

\section{ABSTRACT}

Introduction The majority of patients undergoing haemodialysis (HD) show evidence of uraemic neuropathy, a condition with no known disease-modifying treatments. The pathogenesis of uraemic neuropathy is poorly understood, but may be related to cumulative exposure to middle molecules or other solutes such as potassium. It is not known whether haemodiafiltration (HDF) reduces the progression of uraemic neuropathy.

Methods and analysis Filtration In the Neuropathy of End-Stage kidney disease Symptom Evolution (FINESSE) is a multicentre, randomised, open-label, blinded endpoint assessment, controlled trial designed to assess the impact of HDF versus HD on uraemic neuropathy. Maintenance HD patients will be randomised in a 1:1 ratio to receive HDF or HD with high-flux membranes for 4 years. The primary endpoint is the difference in the mean change in Total Neuropathy Score (TNS) - a measure of peripheral neuropathy combining symptoms, signs and nerve conduction velocity-over the study period. Secondary outcomes include change at annual timepoints in the TNS and the Neuropathy Symptom Score; and in morbidity, mortality and safety events.

Ethics and dissemination The FINESSE trial has been approved by the Ethics Review Committee of the Sydney South West Area Health Service (HREC/09/RPAH/268) and of Adventist HealthCare Limited (2012-027). When published in a peer-reviewed journal, it will be the largest and longest reported randomised trial aimed at reducing the incidence and severity of uraemic neuropathy. It will advance the understanding of the natural history of uraemic neuropathy and the influence of convective therapies on both neurophysiological and clinical outcomes. It will also allow refinement of current hypotheses surrounding the pathogenesis of uraemic neuropathy and, most importantly, may lead to improvements in the lives of the many patients affected by this debilitating condition.

Trial registration number ACTRN12609000615280.

\section{INTRODUCTION}

Worldwide, the number of people with end-stage kidney disease is expected in double by 2030 to more than 5 million, ${ }^{1}$ with most

\section{Strengths and limitations of this study}

- Filtration In the Neuropathy of End-Stage kidney disease Symptom Evolution will be the largest (120 participants) and longest (4 years) study of uraemic neuropathy ever undertaken.

- The primary neuropathy endpoint is assessed by a blinded assessor.

- Participants and caring staff are not blinded.

- The primary neuropathy endpoint is measured using a tool that includes symptoms, signs and nerve conduction measures.

recipients of renal replacement therapy being treated with dialysis. ${ }^{2} 3$ In addition to higher mortality, people receiving maintenance dialysis have greater symptom burden than the general population and lower health-related quality of life (HRQOL). ${ }^{45} \mathrm{~A}$ contributor to the poorer HRQOL in recipients of dialysis is uraemic neuropathy. ${ }^{6}$

Uraemic neuropathy is a common and progressive distal symmetrical polyneuropathy that manifests with the insidious onset of paraesthesia, pain, weakness and muscle wasting. Nerve conduction studies (NCS) are abnormal in 90\%-100\% of patients receiving maintenance dialysis therapy. ${ }^{7}$ The proportion of these who are symptomatic varies widely in published studies, with rates as high as $93 \%$ in small studies, ${ }^{8}$ although the true prevalence of symptomatic uraemic neuropathy may be closer to the $16 \%$ reported in a recent study of 225 prevalent haemodialysis (HD) patients. ${ }^{9} 10$ The pathophysiology of the condition is poorly understood but a causal role has been suggested for middle molecular weight uraemic toxins ('middle molecules') and/or persistent hyperkalaemia. ${ }^{9}$ There are conflicting reports on the impact of improved renal clearance on disease trajectory, with some reports of 
benefit with increased clearance through intensive dialysis ${ }^{11}$ or renal transplantation, ${ }^{12}$ but others of progression or persistence despite transplantation. ${ }^{9} 13$ There are no proven disease-modifying treatments.

Haemodiafiltration (HDF) combines the convective clearance of haemofiltration with HD resulting in enhanced clearance of small and middle molecules, ${ }^{14}$ the most widely measured of which is $\beta 2$-microglobulin. ${ }^{15} 16$ HDF may ameliorate uraemic neuropathy by improved clearance of both middle molecules and smaller uraemic solutes. It has been associated with a reduced incidence of carpal tunnel surgery (possibly suggesting reduced $\beta 2$-microglobulin amyloidosis) ${ }^{17}$ in older reports and with improved nerve excitability measures in the modern era. $^{1819}$

We designed the Filtration In the Neuropathy of End-Stage kidney disease Symptom Evolution (FINESSE) trial to determine the effect of HDF compared with standard high-flux HD on the progression of uraemic neuropathy in recipients of maintenance HD therapy.

\section{METHODS}

\section{Aim and design}

FINESSE is a multicentre, prospective, randomised, openlabel study with blinded endpoint assessment comparing the effect of HDF versus conventional high-flux HD on the incidence and progression of uraemic neuropathy.

\section{Setting and participants}

The study is underway at four dialysis centres (Concord Repatriation General Hospital, Royal Prince Alfred Hospital, Prince of Wales Hospital and Sydney Adventist Hospital) in metropolitan Sydney, Australia. Patients dialysing in-centre, meeting the eligibility criteria (box 1) and able to provide informed consent were invited to participate (figure 1).

\section{Box 1 Inclusion and exclusion criteria}

Inclusion criteria

1. Incident or prevalent patients requiring maintenance haemodialysis therapy for ESKD.

2. Aged 18 years or older.

3. Suitable for either HDF or standard dialysis in the view of the treating physician.

4. Agreeable to randomisation.

Exclusion criteria

1. Life expectancy less than 6 months.

2. Definite plans to undergo renal transplantation, transfer to a nonstudy site, transfer to peritoneal dialysis or transfer to home haemodialysis within 12 months of entry to the study.

3. Receiving HDF.

4. Unable or unwilling to complete neuropathy staging, including nerve conduction studies.

ESKD, end-stage kidney disease; HDF, haemodiafiltration.

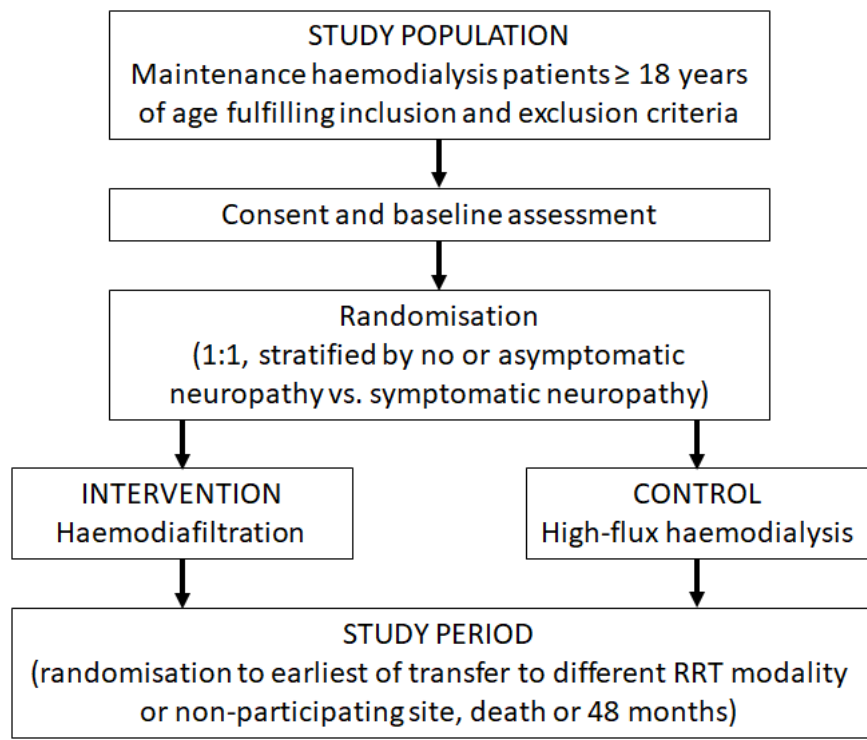

Figure 1 Filtration In the Neuropathy of End-Stage kidney disease Symptom Evolution study flow. RRT, renal replacement therapy.

\section{Study procedures}

Eligible dialysis patients at participating units who provided written informed consent proceeded to the baseline visit. The number of screened patients found to be ineligible or who did not participate and their reasons for non-participation were recorded in the site screening $\log$. Study visits occur at 6-monthly intervals with neurological assessment annually (table 1 ). All participants were asked to consent to follow-up beyond the study period, by direct follow-up until 4 years after the enrolment of the final participant and, separately, via data linkage. Linkage to the Australian and New Zealand Dialysis and Transplant registry (ANZDATA) and to Medicare Australia will enable ascertainment of long-term event endpoints (table 2). While participants and treating clinicians are not blinded, endpoint assessments are undertaken by blinded assessors. Participants are trained not to reveal their allocation to the neurologist/neurophysiologist performing neurological assessment. At the conclusion of the study period, participants and their treating physicians have the option of continuing or altering their allocated treatment.

\section{Intervention and control}

Participants are allocated to HDF or standard HD using a high-flux membrane. While the mode of HDF was not prescribed, predilution HDF was initially used for all participants in the treatment arm until June 2010 when only one site had commenced recruitment. After June 2010, all participants in the treatment arm at all sites received postdilution HDF. No minimum convection volume was mandated, although after the presentation of HDF trials at the European Renal Association-European Dialysis and Transplant Association meeting in June 2011, sites were encouraged to use higher volumes as far as possible. ${ }^{20}$ Background routine medical care includes the 
Table 1 Schedule of visits

\begin{tabular}{|c|c|c|c|c|c|c|c|c|c|c|}
\hline \multirow[b]{2}{*}{ Timepoint } & \multirow[b]{2}{*}{ Baseline } & \multirow[b]{2}{*}{ Randomisation } & \multicolumn{8}{|c|}{ Main study period (months) } \\
\hline & & & 6 & 12 & 18 & 24 & 30 & 36 & 42 & 48 \\
\hline $\begin{array}{l}\text { Consent, } \\
\text { demographics and } \\
\text { dialysis history }\end{array}$ & $x$ & & & & & & & & & \\
\hline $\begin{array}{l}\text { Medications, dialysis } \\
\text { parameters, BP, } \\
\text { laboratory tests }\end{array}$ & $x$ & & $x$ & $x$ & $\mathrm{x}$ & $x$ & $x$ & $x$ & $x$ & $x$ \\
\hline Serum banked & $\mathrm{X}$ & & $X$ & $\mathrm{X}$ & $\mathrm{X}$ & $\mathrm{X}$ & $\mathrm{X}$ & $\mathrm{X}$ & $\mathrm{X}$ & $\mathrm{X}$ \\
\hline $\begin{array}{l}\text { Neurological studies: } \\
\text { NCS, TNS, NSS }\end{array}$ & $x$ & & & $x$ & & $x$ & & $x$ & & $x$ \\
\hline \multicolumn{11}{|l|}{ Subgroup studies } \\
\hline $\begin{array}{l}\text { Nerve excitability } \\
\text { studies }\end{array}$ & $x$ & & & $x$ & & $x$ & & $x$ & & $x$ \\
\hline KDRL Score & $\mathrm{X}$ & & $\mathrm{X}$ & $\mathrm{X}$ & $\mathrm{X}$ & $\mathrm{X}$ & $\mathrm{X}$ & $x$ & $\mathrm{X}$ & $x$ \\
\hline $\mathrm{HbA} 1 c^{*}$ & $\mathrm{X}$ & & & $x$ & & $\mathrm{X}$ & & $x$ & & $x$ \\
\hline
\end{tabular}

${ }^{*}$ Collected only in participants with known diabetes.

BP, blood pressure; EQ5D, EuroQol-5D; HbA1c, glycated haemoglobin; KDQOL-SF, Kidney Disease Quality of Life Short Form; KDRL, Kidney Disease-Related Loss; NCS, nerve conduction studies; NSS, Neuropathy Symptom Score; QOL, Quality of Life; TNS, Total Neuropathy Score.

use of a multivitamin supplement containing water-soluble vitamins (including B-group vitamins) following dialysis. The number of dialysis hours and sessions per week remain at the discretion of the treating physician.

\section{Study duration}

Participants remain in the study for 48 months or until they die, receive a renal transplant, change to peritoneal dialysis, move to a dialysis setting unable to provide both treatments or withdraw consent. Follow-up is expected to average between 30 and 36 months. Recruitment commenced in July 2009 and, due to unforeseen contractual restrictions that allowed only limited access to online HDF, was not completed until late 2013. Data locking is yet to be completed and results are anticipated in 2019 .

\section{Neuropathy assessment}

The primary outcome will be assessed by modified Total Neuropathy Score (TNS). TNS is a measure of peripheral polyneuropathy, is validated in diabetic and chemotherapy-induced neuropathy ${ }^{21}$ and, in line with the consensus statement of the American Academy of Neurology for neuropathy assessment in research, includes symptoms, signs and electrophysiological findings. ${ }^{22}$ It combines a structured symptom questionnaire (0-8 points), neurological examination signs (0-12 points) and tibial and sural nerve sensory amplitudes on NCS ( $0-8$ points), to provide a score of 0 to 28 . Higher scores indicate more severe neuropathy and may be graded on a five-level scale (where grades $0-4$ are, respectively, no, minor, moderate, moderate-severe, severe) (tables 3A, B). TNS includes sural nerve amplitude, which is among the most sensitive NCS parameters for the detection of neuropathy. ${ }^{23-25}$
Reported TNS interexaminer reliability is high (Spearman's rho 0.966).$^{26}$ License for the TNS was provided to Professor Arun Krishnan by Professor David Cornblath and Johns Hopkins University.

An additional assessment tool, the Neuropathy Symptom Score (NSS), is included as a secondary outcome. While it only assesses the symptom domain of neuropathy, NSS is a validated measure devised for diabetic peripheral neuropathy ${ }^{27}$ which has been used in HD populations ${ }^{23}$ and is employed here using the modification described by Krishnan et al (table 4). This questionnaire identifies the presence or absence of symptoms in three categories and assigns one point for each present symptom (four motor, three negative sensory and two positive sensory) with a maximum possible score of 9 points. NSS-based neuropathy stage (table 5 ) will also be calculated by combining the NSS score, NCS results and the presence or absence of 'disabling' neuropathic symptoms (ie, sensory abnormalities).$^{8728}$

Neuropathy assessments are undertaken by qualified neurologists and neurophysiologists under the supervision of Dr Arun Krishnan. Assessors are blinded to participant treatment allocation. Dr Krishnan will duplicate neuropathy assessments performed by each assessor for five separate patients to allow assessment of inter-rater reliability.

\section{Study endpoints}

The primary endpoint is the between-group difference in the mean change in TNS over the study period. The secondary endpoints include a variety of neurological, clinical and safety endpoints (table 2). In addition 
Table 2 Study endpoints

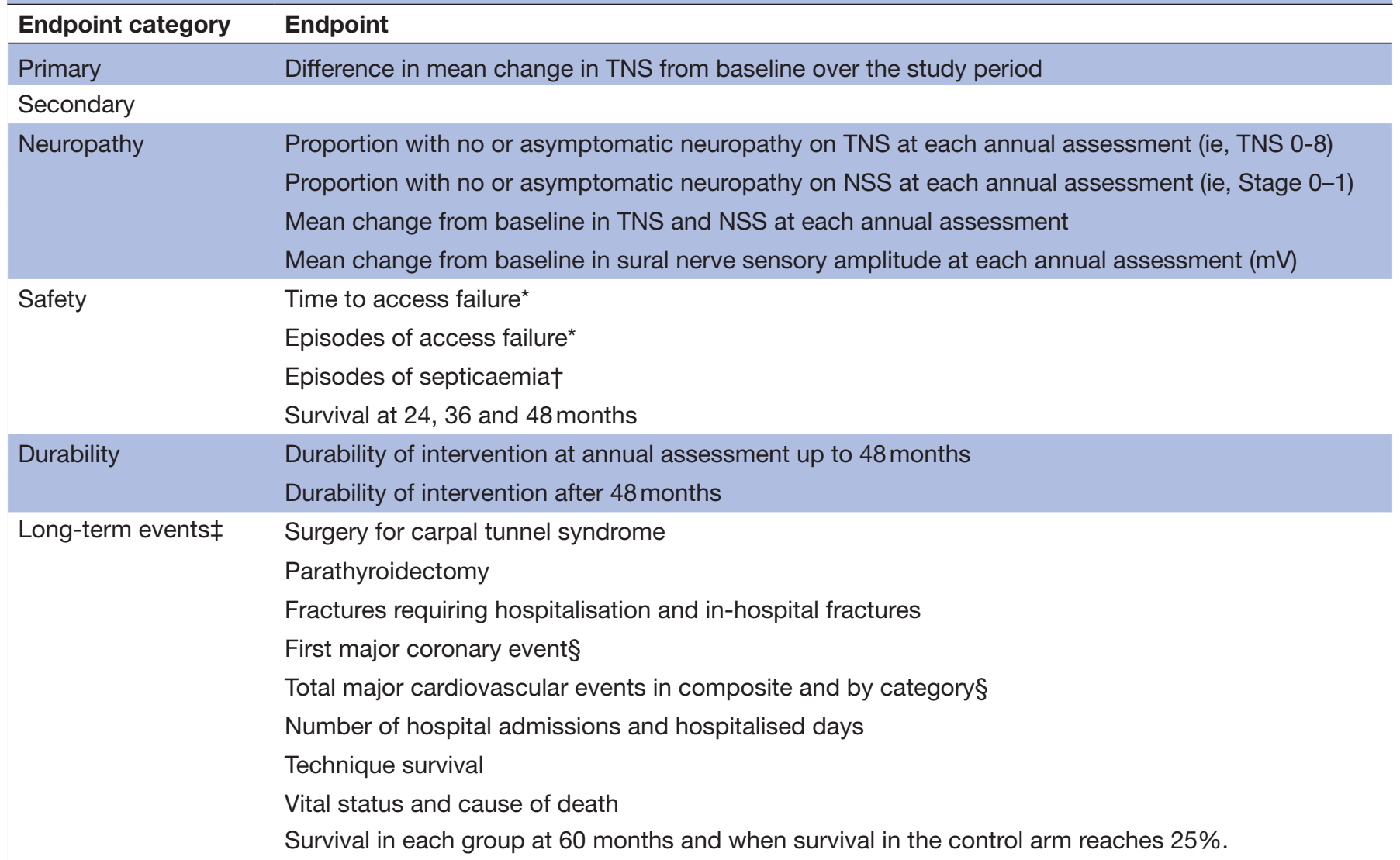

${ }^{*}$ Defined as thrombosis or revision of fistula or graft.

†Defined as blood culture positive septic episode without defined source.

‡Obtained through data linkage, subsequent to the appropriate poststudy approvals.

$\S$ Defined as a composite of cardiovascular death or hospitalisation due to/including any of acute myocardial infarction, cerebrovascular event, percutaneous coronary or cerebrovascular revascularisation or surgical coronary or cerebral revascularisation.

NSS, Neuropathy Symptom Score; TNS, Total Neuropathy Score.

to designated endpoints, all participants have routine biochemical and haematological testing at regular intervals (table 1) including predialysis serum calcium, phosphate, bicarbonate, parathyroid hormone, $\beta 2$-microglobulin, haemoglobin and troponin. A variety of clinical and therapeutic parameters will also be recorded including predialysis systolic blood pressure, dialysis prescription, flow rates, convection volumes and medication use (dose and regimen of phosphate binders, vitamin D analogues, cinacalcet, erythropoietin-stimulating agents and antihypertensive agents).

Participants were also asked to consent to data linkage to the ANZDATA registry and to the Medicare Benefits Schedule and the Pharmaceutical Benefits Scheme records of the Australian universal healthcare system. Data linkage will be used to ascertain vital status, technique survival, healthcare service use and the occurrence of prespecified events during an observational period following the completion of the intervention.

\section{Randomisation}

Participants were randomised in a 1:1 fashion with stratification by baseline neuropathy grade. Strata 1 was defined as TNS grade $0-1$ (no or minor neuropathy) and strata 2 as TNS grade 2-4 (moderate to severe neuropathy) (tables $3 \mathrm{~b}$ ). The allocation sequence was based on blocks of 4 and generated centrally by an independent statistician who had no other involvement in the study. To ensure allocation concealment, randomisation was performed by an independent university employee based on a physically separate site with no other involvement in the study. The randomisation schedule was known only to these two individuals and to an additional independent unblinded statistician who was responsible for preparing reports for the Data Safety Monitoring Board.

\section{Monitoring and safety}

Adverse events and serious adverse events (SAE) are recorded at each study visit. An independent Data Safety Monitoring Board is performing ongoing review of predefined safety parameters and overall study conduct. The committee will autonomously review unblinded data on participant characteristics, including mortality and SAE. 
Table 3A Total Neuropathy Score (TNS)

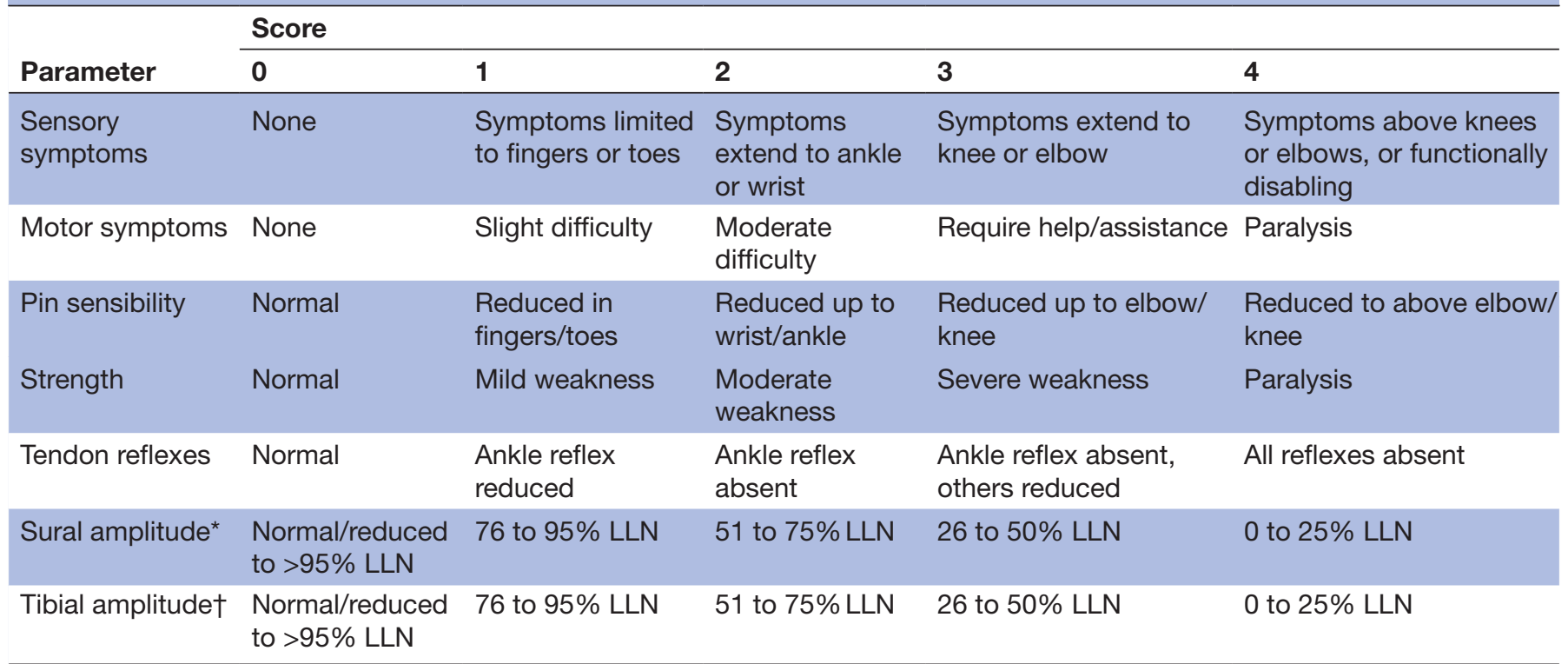

*Lower limit of normal range for sural amplitude by age group (age range (years), amplitude ( $\mu \mathrm{V})$ ): 0-20, $12 \mu \mathrm{V} ; 21-40,9 \mu \mathrm{V} ; 41-60$, $7 \mu \mathrm{V}$; 61-80, $6 \mu \mathrm{V}$.

†Lower limit of normal range for tibial amplitude: $3 \mathrm{mV}$.

\section{Substudies}

Patients who were willing and had sufficient English language ability to self-administer a questionnaire were invited to participate in HRQOL assessments. HRQOL was assessed using the EuroQol-5D-3L, ${ }^{29}$ Kidney Disease Quality of Life Short Form V.1.3 ${ }^{30}$ and the Kidney Disease-Related Loss Score. ${ }^{31}$

\section{Study power}

TNS score was chosen as the primary outcome based on the recommendations of the American Academy of Neurology, which strongly emphasised the need for composite measures that incorporated symptomatic and nerve conduction assessments. ${ }^{22}$ However, at the time of study design, no existing reports for composite score measurements in dialysis patients were available to inform study power calculations. The study was thus powered for the proportion of patients with symptomatic neuropathy as this data was available and constitutes an important secondary outcome. The expected prevalence of TNS grades in the control group was derived from published data using NSS. ${ }^{7823}$ The recruitment target of 120 participants was determined as the sample size that provided

\begin{tabular}{llcl}
\hline \multicolumn{3}{l}{ Table 3B } & Severity grade for TNS \\
\hline $\begin{array}{l}\text { Symptom } \\
\text { status }\end{array}$ & Grade & Score & $\begin{array}{l}\text { Descriptive } \\
\text { terminology }\end{array}$ \\
\hline Asymptomatic & 0 & $0-1$ & None \\
& 1 & $2-8$ & Minor \\
Symptomatic & 2 & $9-16$ & Moderate \\
& 3 & $17-24$ & Moderately severe \\
& 4 & $25-28$ & Severe \\
\hline
\end{tabular}

$90 \%$ power (alpha $=0.05$ ) to detect a reduction in the prevalence of moderate to severe neuropathy from $80 \%$ in the control arm to $48 \%$ in the treatment arm (absolute difference of $32 \%$ ), including an allowance for $20 \%$ combined dropout and loss to follow-up ( $n=96$ in the final analysis). In addition, we also calculated that with $90 \%$ power (with alpha $=0.05$ ) the study could detect an absolute difference of $2.56 \mu \mathrm{V}$ in the mean response of sural nerve sensory amplitudes between the treatment groups (assuming a mean sural nerve sensory amplitude of

Table 4 Modified Neuropathy Symptom Score

\section{Score 1 point for presence of a symptom}

Symptoms of muscle weakness

Symptoms of limb Shoulder girdle and upper arm muscle weakness

$\begin{array}{ll} & \text { Hand } \\ \text { Glutei and thigh } \\ \text { Legs }\end{array}$

Sensory disturbances

\begin{tabular}{ll}
$\begin{array}{l}\text { Negative } \\
\text { symptoms }\end{array}$ & Difficulty identifying objects in mouth \\
& Difficulty identifying objects in hands \\
& Unsteadiness in walking \\
Positive symptoms & $\begin{array}{l}\text { 'Numbness,' 'part of your body is } \\
\text { asleep,' 'like having been given local } \\
\text { anaesthetic,' 'pins and needles', } \\
\text { 'prickling,'-at any site } \\
\text { Pain-burning, deep aching, } \\
\text { tenderness - at any location }\end{array}$ \\
\hline
\end{tabular}


Table 5 Neuropathy Symptom Score (NSS) stages

\section{Definition}

\begin{tabular}{llll}
\cline { 2 - 4 } Stage & $\begin{array}{l}\text { NSS score } \\
\text { (max 9) }\end{array}$ & NCS & $\begin{array}{l}\text { 'Disabling' } \\
\text { neuropathic } \\
\text { symptoms }\end{array}$ \\
\hline $\begin{array}{l}\text { No neuropathy } \\
\text { Non }\end{array}$ & $<2$ & Normal & No \\
1 & 0 & Abnormal & No \\
Asymptomatic & & Normal & No \\
2 & $\geq 2$ & Abnormal & No \\
Symptomatic & $\geq 1$ & Normal & Yes \\
3 & $\geq 2$ & Abnormal & Yes \\
\hline
\end{tabular}

Adapted from Krishnan et al. ${ }^{28}$

NCS, nerve conduction studies.

$5.8 \pm 3.9 \mu \mathrm{V})$. More recently, studies have been conducted that provide data on the TNS from an external cohort (Krishnan A, personal communication). In 49 dialysis patients, the mean TNS was $9.2 \pm 7.8$ and $42 \%$ (20/48) had TNS grade 2-4 (ie, moderate to severe). Using these assumptions, FINESSE has $90 \%$ power to detect a mean difference of 5.2 in TNS between treatment arms at study end and $80 \%$ power to detect a mean difference of 4.5 . A minimum clinically meaningful difference in neuropathy has been defined as a difference in lower limb motor nerve conduction velocity of $2.2 \mathrm{~m} / \mathrm{s},{ }^{32}$ or a change of 2 points in a standardised clinical examination ${ }^{33-}$ both of which individually result in a change of 2 in TNS score.

\section{Statistical analysis}

All analyses will be performed on an intention-to-treat basis. In the primary analysis, the mean change in TNS from baseline will be analysed using a restricted maximum likelihood-based repeated measures approach. Analyses will include the fixed, categorical effects of treatment, visit and treatment-by-visit interaction, as well as the continuous, fixed covariates of baseline score and baseline score-by-visit interaction. Outside of the primary analysis, binary endpoints will be analysed using the $\chi^{2}$ test to compare proportions. Mann-Whitney U test or transformations may be used when distributions are skewed or not normally distributed. Odds ratios will be estimated using logistic regression analysis. For continuous repeated measures, secondary analysis will be performed using a linear mixed model including random intercept, randomisation and time categories.

\section{Ethics and dissemination}

The study received ethical approval. The study is overseen by an independent Data Safety Monitoring Board, coordinated by the Australasian Kidney Trials Network. The FINESSE Study is registered with the Australian New Zealand Clinical Trials Registry (ACTRN12609000615280). The results are intended to be disseminated through conference presentations and publication in the peer-reviewed medical literature.

\section{Patient and public involvement statement}

The study objective was to assess the impact of two dialysis modalities in current practice on the severity of neuropathy as assessed in part by patient-reported symptoms and function. There was no direct patient or public contribution to the study design.

\section{DISCUSSION}

FINESSE is the largest and longest randomised trial aiming to test a disease-modifying intervention for uraemic neuropathy. This unique cohort of patients will provide valuable information on the natural history of uraemic neuropathy and will determine the place of HDF in its management. As the advent of online ultrapure dialysate generation has reduced the cost of delivering HDF, any benefits shown in FINESSE will be implementable with only a modest cost increment and no requirement for increase in pill burden or time on dialysis.

The primary cause of uraemic neuropathy is believed to be middle molecular weight uraemic toxins ('middle molecules', defined as uraemic toxins with a mass of 500-60 000 Da). Support for this hypothesis comes from the decline in the prevalence of $\beta 2$-microglobulin amyloidosis and severe uraemic neuropathy correlating with the widespread adoption of high-flux dialysis membranes (defined by their greater clearance of middle molecules).$^{34}$ Prior to the introduction of high-flux membranes for HD, peritoneal dialysis patients had a lower prevalence of uraemic neuropathy and recent evidence suggests that peritoneal dialysis may be associated with better neurophysiological parameters than HD, differences attributed to the greater middle molecule clearance afforded by the peritoneal membrane. ${ }^{35} 36$ In addition, residual renal function also provides increased middle molecule clearance and is inversely correlated with the presence of neurophysiological abnormalities. ${ }^{37}$ Despite these associations, prospective evidence for the middle molecule hypothesis is scarce. Dramatic improvements were shown in an early study using a membrane highly permeable to middle molecules ${ }^{35}$ and a recently published trial of 66 participants randomised to HD or haemoperfusion plus HD over 12 weeks reported an increase in sensory conduction velocity and an improvement in symptoms that correlated with a reduction in $\beta 2$-microglobulin. ${ }^{38}$ However, large-scale prospective dialysis trials have not included neuropathy as an outcome.

Despite a large body of circumstantial and observational evidence, no single solute in the middle molecular range has been convincingly identified as the culprit neurotoxin. ${ }^{9}$ In response to this, an alternative hypothesis attributes a pathogenic role to persistent hyperkalaemia, which is common in HD patients and may lead to axonal loss via chronic activation of damaging calcium-dependent intracellular mechanisms. Predialysis hyperkalaemia 
has been shown to cause peripheral nerve depolarisation which improves in the immediate postdialysis period. ${ }^{8}$ Dietary potassium restriction has recently been shown to prevent deterioration in uraemic neuropathy in a small, randomised trial in patients with non-dialysis-dependent chronic kidney disease. ${ }^{39}$ While the effect of HDF on middle molecule clearance is the most pronounced difference from conventional HD, HDF does also improve the clearance of small molecules such as potassium. ${ }^{14}$ Thus, HDF may plausibly improve uraemic neuropathy outcomes regardless of the underlying pathophysiological mechanisms.

The natural history of uraemic neuropathy also remains incompletely understood. Subclinical disease (abnormal electrophysiological studies in the absence of symptoms) is almost universal, being present in $60 \%-100 \%$ of patients. ${ }^{9}$ However, it is not clear what proportion of asymptomatic patients will progress to experience symptoms or whether established disease progresses in the face of adequate dialysis. Cross-sectional studies suggest that electrophysiological findings are worse in patients who have been on dialysis for a longer period, and that both clinical and electrophysiological findings increase with patient age. ${ }^{24} 40$ The severity of uraemic neuropathy on a single assessment has been associated with mortality. ${ }^{10} 41$ As a prospective, longitudinal study, the current trial affords the opportunity to clarify important aspects of the natural history of uraemic neuropathy, and may uncover additional modifiable and non-modifiable risk factors for disease progression.

While pilot studies have demonstrated improvement in symptoms and NCS parameters with zinc supplementation of dialysate, with vitamin B12 and with erythropoietin therapy, ${ }^{42-44}$ there is no established disease-altering treatment for uraemic neuropathy. Moreover, even the therapeutic effect of dialysis on uraemic neuropathy is unclear. Adequate peritoneal and HD appear to be equally effective in retarding the progression of established uraemic neuropathy but neither is likely to lead to significant improvement. ${ }^{45}$ Case reports suggest that improvement in symptoms and neurophysiological abnormalities can be associated with intensive HD (5-6 times per week $)^{47}$ while the association with renal transplantation is the subject of conflicting reports. ${ }^{12} 48$ Even if intensive HD and transplantation are effective treatments, they may not be available modalities for all, especially the very frail. HDF is a modality that is more widely accessible and can be delivered with minimal, if any, increase in cost. ${ }^{49}$

FINESSE trial will also provide a deeper understanding of the utility of the clinical measures of uraemic neuropathy. While NCS measures are only loosely correlated with symptoms and signs of uraemic neuropathy, the multimodal TNS permits the identification of patients with the full range of symptoms, signs and electrophysiological abnormalities. However, the minimum clinically meaningful difference for TNS is not known. This study may permit a greater understanding of the meaning of this measure in accordance with the growing focus on patient-centred outcomes in clinical research. ${ }^{50}$

In conclusion, morbidity due to uraemic neuropathy is expected to increase in line with the increases in dialysis numbers, proportion of patients with concurrent diabetes and waiting time for kidney transplantation. FINESSE trial will provide a new perspective on uraemic neuropathy in the modern era and add substantially to current understanding of the benefits of HDF.

\section{Author affiliations}

${ }^{1}$ Renal and Metabolic, The George Institute for Global Health, UNSW, Newtown, New South Wales, Australia

${ }^{2}$ Neurology, Prince of Wales Clinical School, UNSW, Sydney, New South Wales, Australia

${ }^{3}$ Renal Medicine, Concord Repatriation General Hospital, Sydney, New South Wales, Australia

${ }^{4}$ Brain and Mind Centre, University of Sydney, Sydney, New South Wales, Australia ${ }^{5}$ Renal Medicine, Royal Prince Alfred Hospital, Sydney, New South Wales, Australia ${ }^{6}$ Renal Medicine, Princess Alexandra Hospital, Brisbane, Queensland, Australia ${ }^{7}$ Renal Medicine, Sydney Adventist Hospital, Sydney, New South Wales, Australia ${ }^{9}$ Renal Medicine, Prince of Wales Hospital, Sydney, New South Wales, Australia

Contributors BS contributed to the analysis and interpretation, and drafted the manuscript. MG, CH, AVK, MK and VP are Trial Steering Committee members, contributed to the study design, and oversaw the conduct of the study. MF and PS are Trial Steering Committee members, and oversaw the conduct of the study. SH, $\mathrm{KG}, \mathrm{JB}, \mathrm{AH}$ and $\mathrm{AK}$ contributed to the acquisition of data. MJJ, Chair of the Trial Steering Committee, is primarily responsible for the conception and design of the study and oversees the conduct of the study.

Funding The study was funded by a Jacquot Research Establishment Award administered by the Royal Australian College of Physicians in 2011 and 2012, and by an unrestricted grant from Baxter Healthcare Pty Ltd. The purchase of two nerve conduction machines was supported by an unrestricted grant from the Royal Prince Alfred Hospital Renal Unit Trust and from Shire Pharmaceuticals. BS is supported by an Australian Government Research Training Program Scholarship via the University of Sydney. MK is funded by Forefront, a collaborative research group supported by National Health and Medical Research Council of Australia (NHMRC) Program Grant (\#1037746). AK is supported by a NHMRC Postgraduate Scholarship via the University of New South Wales, an Australian Government Research Training Program Fees Offset and has received a George Institute Scholarship. MJ is funded by a Medical Research Future Fund Career Development Fellowship.

Competing interests MJ serves on an advisory board for Baxter Healthcare.

Patient consent for publication Not required.

Ethics approval Ethics Review Committee (Royal Prince Alfred Hospital (RPAH) Zone) of the Sydney South West Area Health Service (HREC/09/RPAH/268)

Provenance and peer review Not commissioned; externally peer reviewed.

Open access This is an open access article distributed in accordance with the Creative Commons Attribution Non Commercial (CC BY-NC 4.0) license, which permits others to distribute, remix, adapt, build upon this work non-commercially, and license their derivative works on different terms, provided the original work is properly cited, appropriate credit is given, any changes made indicated, and the use is non-commercial. See: http://creativecommons.org/licenses/by-nc/4.0/.

\section{REFERENCES}

1. Liyanage T, Ninomiya T, Jha V, et al. Worldwide access to treatment for end-stage kidney disease: a systematic review. Lancet 2015;385:1975-82.

2. Wolfe RA, Ashby VB, Milford EL, et al. Comparison of mortality in all patients on dialysis, patients on dialysis awaiting transplantation, and recipients of a first cadaveric transplant. $N$ Engl J Med 1999;341:1725-30.

3. Shafran D, Kodish E, Tzakis A. Organ shortage: the greatest challenge facing transplant medicine. World J Surg 2014;38:1650-7.

4. Valderrábano F, Jofre R, López-Gómez JM. Quality of life in endstage renal disease patients. Am J Kidney Dis 2001;38:443-64. 
5. Murtagh FE, Addington-Hall J, Higginson IJ. The prevalence of symptoms in end-stage renal disease: a systematic review. Adv Chronic Kidney Dis 2007;14:82-99.

6. Santoro D, Satta E, Messina S, et al. Pain in end-stage renal disease: a frequent and neglected clinical problem. Clin Nephrol 2013;79 Suppl 1(Suppl 1):S2-11.

7. Tilki HE, Akpolat T, Coșkun M, et al. Clinical and electrophysiologic findings in dialysis patients. J Electromyogr Kinesiol 2009;19:500-8.

8. Krishnan AV, Phoon RK, Pussell BA, et al. Altered motor nerve excitability in end-stage kidney disease. Brain 2005;128(Pt 9):2164-74

9. Krishnan AV, Kiernan MC. Uremic neuropathy: clinical features and new pathophysiological insights. Muscle Nerve 2007;35:273-90.

10. Mambelli E, Barrella M, Facchini MG, et al. The prevalence of peripheral neuropathy in hemodialysis patients. Clin Nephrol 2012;77:468-75

11. Deger SM, Reis KA, Guz G, et al. A case of an accelerated uremic neuropathy. Ren Fail 2011;33:371-2.

12. Hupperts RM, Leunissen KM, van Hooff JP, et al. Recovery of uremic neuropathy after renal transplantation. Clin Neurol Neurosurg 1990;92:87-9.

13. Arnold R, Pussell BA, Pianta TJ, et al. Association between calcineurin inhibitor treatment and peripheral nerve dysfunction in renal transplant recipients. Am J Transplant 2013;13:2426-32.

14. Wang AY, Ninomiya T, Al-Kahwa A, et al. Effect of hemodiafiltration or hemofiltration compared with hemodialysis on mortality and cardiovascular disease in chronic kidney failure: a systematic review and meta-analysis of randomized trials. Am J Kidney Dis 2014;63:968-78.

15. Wizemann V, Lotz C, Techert F, et al. On-line haemodiafiltration versus low-flux haemodialysis. A prospective randomized study. Nephrol Dial Transplant 2000;15 Suppl 1(Suppl 1):43-8.

16. Schiffl $H$. Prospective randomized cross-over long-term comparison of online haemodiafiltration and ultrapure high-flux haemodialysis. Eur J Med Res 2007;12:26-33.

17. Locatelli F, Marcelli D, Conte F, et al. Comparison of mortality in ESRD patients on convective and diffusive extracorporeal treatments. The Registro Lombardo Dialisi E Trapianto. Kidney Int 1999;55:286-93.

18. Arnold R, Pussell BA, Pianta TJ, et al. Effects of hemodiafiltration and high flux hemodialysis on nerve excitability in end-stage kidney disease. PLoS One 2013;8:e59055.

19. Malberti F, Surian M, Farina M, et al. Effect of hemodialysis and hemodiafiltration on uremic neuropathy. Blood Purif 1991;9(56):285-95.

20. Ok E, Asci G, Es O, et al. Comparison of post-dilution on-line hemodiafiltration and hemodialysis (Turkish HDF Study) [Abstracts from the 48th ERA-EDTA Congress, June 23-26 2011, Prague, Czech Republic]. NDT Plus 2011;4.4.s2.1.

21. Issar T, Arnold R, Kwai NCG, et al. The utility of the Total Neuropathy Score as an instrument to assess neuropathy severity in chronic kidney disease: A validation study. Clin Neurophysiol 2018;129:889-94.

22. England JD, Gronseth GS, Franklin G, et al. Distal symmetric polyneuropathy: a definition for clinical research: report of the American Academy of Neurology, the American Association of Electrodiagnostic Medicine, and the American Academy of Physical Medicine and Rehabilitation. Neurology 2005;64:199-207.

23. Laaksonen S, Metsärinne K, Voipio-Pulkki LM, et al. Neurophysiologic parameters and symptoms in chronic renal failure. Muscle Nerve 2002;25:884-90.

24. Jurcić D, Bilić $A$, Schwarz D, et al. Clinical course of uremic neuropathy in long-term hemodialysis. Coll Antropol 2008;32:771-5.

25. Bazzi C, Pagani C, Sorgato G, et al. Uremic polyneuropathy: a clinical and electrophysiological study in 135 short- and long-term hemodialyzed patients. Clin Nephrol 1991;35:176-81.

26. Cornblath DR, Chaudhry V, Carter K, et al. Total neuropathy score: validation and reliability study. Neurology 1999;53:1660.
27. Dyck PJ, Detection DPJ. Detection, characterization, and staging of polyneuropathy: assessed in diabetics. Muscle Nerve 1988;11:21-32.

28. Krishnan AV, Phoon RK, Pussell BA, et al. Sensory nerve excitability and neuropathy in end stage kidney disease. J Neurol Neurosurg Psychiatry 2006;77:548-51.

29. Rabin R, de Charro F. EQ-5D: a measure of health status from the EuroQol Group. Ann Med 2001;33:337-43.

30. Hays R, Kallich J, Mapes D, et al. Kidney Disease Quality of Life Short Form (KDQOL-SFTM), Version 1.3: A Manual for Use and Scoring. Santa Monica, CA: RANDP-, 19947994.

31. Chan R, Brooks R, Gallagher M, et al. Measuring kidney diseaserelated loss in samples of predialysis and dialysis patients: validating the kidney disease loss scale. Clin J Am Soc Nephrol 2010;5:1249-54.

32. Dyck PJ, O'Brien PC. Meaningful degrees of prevention or improvement of nerve conduction in controlled clinical trials of diabetic neuropathy. Diabetes Care 1989;12:649-52.

33. Bril V. NIS-LL: the primary measurement scale for clinical trial endpoints in diabetic peripheral neuropathy. Eur Neurol 1999;41 Suppl 1(Suppl 1):8-13.

34. Floege J, Ketteler M. beta2-microglobulin-derived amyloidosis: an update. Kidney Int Suppl 2001;78:164-71.

35. Vanholder R, De Smet R, Hsu C, et al. Uremic toxicity: the middle molecule hypothesis revisited. Semin Nephrol 1994;14:205-18.

36. Arnold R, Pussell BA, Kiernan MC, et al. Comparative study to evaluate the effects of peritoneal and hemodialysis on peripheral nerve function. Muscle Nerve 2016;54:58-64.

37. Janda K, Stompór T, Gryz E, et al. [Evaluation of polyneuropathy severity in chronic renal failure patients on continuous ambulatory peritoneal dialysis or on maintenance hemodialysis]. Przegl Lek 2007;64:423-30.

38. Cai W, Miao S, Wang P, et al. [Efficacy and mechanism of hemoperfusion plus hemodialysis for peripheral neuropathy of uremic patients on maintenance hemodialysis]. Zhonghua Yi Xue Za Zhi 2015;95:1319-22.

39. Arnold R, Pianta TJ, Pussell BA, et al. Randomized, controlled trial of the effect of dietary potassium restriction on nerve function in CKD. Clin J Am Soc Nephrol 2017;12:1569-77.

40. Matsumoto H, Saito K, Konoma Y, et al. Motor cortical excitability in peritoneal dialysis: a single-pulse TMS study. J Physiol Sci 2015;65:113-9.

41. Stosovic M, Nikolic A, Stanojevic M, et al. Nerve conduction studies and prediction of mortality in hemodialysis patients. Ren Fail 2008;30:695-9.

42. Hassan K, Simri W, Rubenchik I, et al. Effect of erythropoietin therapy on polyneuropathy in predialytic patients. $J$ Nephrol 2003;16:121-5.

43. Sprenger KB, Bundschu D, Lewis K, et al. Improvement of uremic neuropathy and hypogeusia by dialysate zinc supplementation: a double-blind study. Kidney Int Suppl 1983;16:S315-S18.

44. Kuwabara S, Nakazawa R, Azuma N, et al. Intravenous methylcobalamin treatment for uremic and diabetic neuropathy in chronic hemodialysis patients. Intern Med 1999;38:472-5.

45. Ogura T, Makinodan A, Kubo T, et al. Electrophysiological course of uraemic neuropathy in haemodialysis patients. Postgrad Med J 2001;77:451-4.

46. Tegnér R, Lindholm B. Uremic polyneuropathy: different effects of hemodialysis and continuous ambulatory peritoneal dialysis. Acta Med Scand 1985;218:409-16.

47. Ghazan-Shahi S, Koh TJ, Chan CT. Impact of nocturnal hemodialysis on peripheral uremic neuropathy. BMC Nephrol 2015;16:134-7.

48. Ibrahim MM, Barnes AD, Crosland JM, et al. Effect of renal transplantation of uraemic neuropathy. Lancet 1974;2:739-42.

49. Oates T, Cross J, Davenport A. Cost comparison of online haemodiafiltration with high-flux haemodialysis. J Nephrol 2012;25:192-7.

50. Tong A, Manns B, Hemmelgarn B, et al. Standardised outcomes in nephrology - Haemodialysis (SONG-HD): study protocol for establishing a core outcome set in haemodialysis. Trials 2015;16:364. 